\title{
Rare Occurrence of Pseudomyxoma Peritonei (PMP) Syndrome Arising from a Malignant Transformed Ovarian Primary Mature Cystic Teratoma Treated by Cytoreductive Surgery and HIPEC: A Case Report
}

\section{Francesca M. Ponzini}

Sidney Kimmel Medical College at Thomas Jefferson University, Philadelphia, PA.

\section{Luke L. Kowal}

Sidney Kimmel Medical College at Thomas Jefferson University, Philadelphia, PA.

Mariam Ghafoor

Department of Pathology, Thomas Jefferson University Hospital, Philadelphia, PA.

\section{Allison Goldberg}

Department of Pathology, Thomas Jefferson University Hospital, Philadelphia, PA.

Joanna Chan

Department of Pathology, Thomas Jefferson University Hospital, Philadelphia, PA.

Ryan Lamm

Department of Surgery, Thomas Jefferson University Hospital, Philadelphia, PA.

\section{Shawnna M. Cannaday}

Department of Surgery, Thomas Jefferson University Hospital, Philadelphia, PA.

Scott D. Richard

Department of Surgery, Thomas Jefferson University Hospital, Philadelphia, PA.

\section{Avinoam Nevler}

Department of Surgery, Thomas Jefferson University Hospital, Philadelphia, PA.

\section{Harish Lavu}

Department of Surgery, Thomas Jefferson University Hospital, Philadelphia, PA.

\section{Wilbur B. Bowne ( $\sim$ Wilbur.Bowne@jefferson.edu )}

Department of Surgery, Thomas Jefferson University Hospital, Philadelphia, PA.

\section{Norman G. Rosenblum}

Division of Gynecologic Oncology, Department of Obstetrics and Gynecology, Thomas Jefferson University Hospital, Philadelphia, PA. 
Keywords: pseudomyxoma peritonei, ovarian, teratoma, appendiceal mucocele, disseminated peritoneal adenomucinosis, hyperthermic intraperitoneal chemotherapy (HIPEC), cytoreductive surgery (CRS)

Posted Date: February 22nd, 2022

DOI: https://doi.org/10.21203/rs.3.rs-1374838/v1

License: (c) (1) This work is licensed under a Creative Commons Attribution 4.0 International License. Read Full License

Version of Record: A version of this preprint was published at World Journal of Surgical Oncology on March 11th, 2022. See the published version at https://doi.org/10.1186/s12957-022-02548-8. 


\section{Abstract}

Background: Pseudomyxoma peritonei (PMP) syndrome is a disease process that typically occurs from ruptured appendiceal mucocele neoplasms. PMP syndrome arising from malignant transformation of an ovarian primary mature cystic teratoma (MCT) is a pathogenesis rarely encountered.

Case Presentation: Herein, we report a twenty-eight-year-old patient evaluated and treated for a right ovarian mass and large volume symptomatic abdominopelvic mucinous ascites. Molecular profiling and genetic analysis revealed mutations in ATM, GNAS, and KRAS proteins while IHC demonstrated gastrointestinal specific staining for CK20, CDX2, CK7 and SATB2. Peritoneal cytology showed paucicellular mucin. Diffuse peritoneal adenomucinosis (DPAM) variant of PMP arising from a ruptured ovarian primary MCT after malignant transformation to a low grade appendiceal-like mucinous neoplasm was ultimately confirmed. Treatment included staged therapeutic tumor debulking and right salpingooophorectomy followed by cytoreductive surgery and hyperthermic intraperitoneal chemotherapy (HIPEC).

Conclusions: Our report builds upon the existing literature supporting this aggressive treatment option reserved for advanced abdominal malignancies utilized in this patient with a rare clinical entity.

\section{Introduction}

Pseudomyxoma peritonei (PMP) is a rare condition associated with ruptured mucinous neoplasms that typically originate from the appendix. PMP arising from malignant transformation of an ovarian teratoma is exceedingly rare with a reported incidence of 1-2/1,000,000 per year. ${ }^{1}$ PMP syndrome presents with a variable spectrum of disease biology and malignant potential but commonly results in widespread mucinous ascites disseminated throughout the abdomen and pelvis. ${ }^{2,3}$ If left untreated, patient demise occurs from mass effect of large accumulations of mucinous ascites and tumor causing bowel obstruction, perforation and related sequela. ${ }^{4}$ Computed tomography (CT), magnetic resonance imaging (MRI) and expert pathologic review are mandatory to establish PMP diagnosis prior to commencing with treatment. $^{5}$

Definitive treatment for PMP is complete gross resection and/or cytoreductive surgical (CRS) removal of all visible macroscopic tumor burden followed by hyperthermic intraperitoneal chemotherapy (HIPEC) ${ }^{5,6}$ CRS and HIPEC is a complex surgical procedure that requires a multidisciplinary team approach. ${ }^{7}$ At our institution, a specialized team of surgeons routinely provides this treatment in our appendix derived PMP patients with optimal oncologic outcomes and low morbidity. ${ }^{8}$

In patients with ovaries, determining origin of PMP is challenging when a non-appendiceal source is suspected, implicating the possibility of a rare ovarian mucinous tumor etiology. Historically, Ronnett et al. classified PMP as being ovarian in origin if the appendix cannot be identified and/or if rupture of a mucinous tumor of the appendix goes undetected. ${ }^{2}$ 
Herein, we describe a very rare occurrence of PMP syndrome arising from a ruptured low grade appendiceal-like mucinous neoplasm that evolved from a transformed ovarian primary mature cystic teratoma (MCT) in a young patient that was definitively treated by cytoreductive surgery and regional intraperitoneal perfusion with hyperthermic chemotherapy.

\section{Case Report}

A twenty-eight-year-old female presented to the emergency department for a gynecological consultation after imaging performed at an outside hospital showed a large right ovarian mass concerning for malignancy. The patient's presentation followed two months of upper abdominal pain aggravated by movement, coincident with weight loss, shortness of breath, abdominal distention and decreased appetite. Her ultrasound and CT scan showed an $18 \mathrm{~cm}$ abdominopelvic mass originating from the right ovary which was cystic in appearance containing soft tissue, fatty and calcified components. Importantly, there was large volume symptomatic mucinous appearing ascites (Figure 1).

Serologic tumor markers including CA-125, CA19-9, and CEA, were normal. Past medical, surgical, social, and family history were non-contributory.

The patient underwent a two-staged operative approach. The index procedure included debulking of symptomatic mucinous ascites and resection of a pelvic mass requiring a right salpingo-oophorectomy. An $18 \mathrm{~cm}$ right ovarian tumor was described within a large volume of mucinous ascites. Pathology review demonstrated a mature cystic teratoma containing a mucinous epithelial component (Figure 2).

The ovarian mass contained multiple thick mucoid fluid-filled cysts ranging in size from 3 to $15 \mathrm{~cm}$. In addition, the mass appeared grossly necrotic with soft tan-gray areas interspersed amongst follicles of mature hair. The fallopian tube was not involved. Neoplastic cells on immunohistochemistry (IHC) were strongly positive for CK20, CDX2, and SATB2, and focally positive for CK7 (Figure 2).

Figure 2. A: Mucinous neoplasm, right ovary, showing complex mucinous epithelium (200x); B: Hair follicles (red arrow) with adjacent mucinous neoplasm (40x); C. SATB2 showing strong nuclear staining (40x); D: CDX2/CK20 dual stain showing strong nuclear and cytoplasmic staining, respectively (40x); E. CK7 showing focal cytoplasmic staining.

Pelvic washings revealed abundant extracellular mucin and scant reactive mesothelial cells. Peritoneal cytology demonstrated mucicarmine (mucin) staining of the dense ascites. These clinical and pathologic findings were consistent with the diffuse peritoneal adenomucinosis (DPAM) variant of PMP originating from a ruptured appendiceal-like tissue arising from a malignant transformation of an ovarian mature cystic teratoma.

After multidisciplinary review, completion of CRS and HIPEC was recommended. For the HIPEC portion of the procedure, $40 \mathrm{mgs}$ of alkylating agent mitomycin-C (MMC) was employed. The peritoneal cancer index (PCI) was scored and calculated as described by Jacquet and Sugarbaker. ${ }^{9}$ Calculated total PCI was 21. 
A complete macroscopic cytoreduction of all disease was achieved. Procedures included a greater and lesser omentectomy, omental bursectomy, splenectomy, cholecystectomy, right and left upper quadrant and subhepatic parietal peritonectomies, partial glissonian capsulectomy, appendectomy, removal of small bowel mesenteric and serosal implants, total abdominal hysterectomy, left salpingo-oophorectomy, and pelvic peritonectomy with cytoreduction of mucinous peritoneal disease implants including resection of the upper vagina.

Pathology after complete macroscopic cytoreduction similarly demonstrated abundant pools of paucicellular mucin on the resected abdominopelvic disease-bearing visceral and parietal peritoneal surfaces. Importantly, the appendix was diagnosed as benign on permanent histology. Abundant pools of acellular mucin demonstrated no additional or synchronous malignancy and was confirmatory for the DPAM-variant of PMP. Outpatient genetic counseling revealed no familial and/or genetic predisposition.

The patient's tumor was sent for molecular profiling and next generation sequencing. The genetic report showed mutations in ATM at splice site 8268+1G>A, GNAS R201C, and KRAS G12D. The molecular profile was also consistent with tumor-associated microsatellite stability with a tumor mutational burden of 3Muts/Mb. Due to the low-grade nature of the tumor, systemic therapy was not recommended. The patient will undergo a biannual routine examination and cross-sectional imaging. Currently there is no evidence of disease recurrence one year after the procedure.

\section{Discussion}

Mature cystic teratomas (MCTs) otherwise known as dermoid cysts are benign and frequently present as ovarian neoplasms. However, in $0.2-2 \%$ of cases, they can undergo malignant transformation, as described in this patient. ${ }^{10}$ The majority of these transformations develop in post-menopausal patients between the ages of 50-70 years with squamous cell tumorigenesis, most commonly reported. ${ }^{10}$ Our patient presented at age 28 years. ${ }^{11}$ MCTs reportedly can also undergo transformation to adenocarcinomas, basal cell carcinomas, melanoma, and neuroendocrine tumors due to the malignant potential of pluripotent cells inherent in these tumors (Table 1). 
Table 1

Prevalence of malignant transformations of mature cystic teratomas

\begin{tabular}{|ll|}
\hline Histopathological Type & Prevalence (\% of MT) \\
\hline Squamous Cell Carcinoma & $80^{20}$ \\
\hline Adenocarcinoma & $5^{20}$ \\
\hline Transitional Cell Carcinoma & $<1^{21}$ \\
\hline Malignant Melanoma & $0.2-0.8^{22}$ \\
\hline Thyroid Carcinoma & $0.1-0.2^{23}$ \\
\hline Carcinoid Tumor & $<0.1^{24}$ \\
\hline
\end{tabular}

PMP syndrome typically arises from perforation and/or rupture of an appendiceal mucocele or low grade appendiceal mucocele neoplasms (LAMN). ${ }^{12,13}$ Extra-appendiceal etiologies, although rare, can be ovarian in origin. ${ }^{2,3}$ High clinical suspicion and evidence supporting an extra-appendiceal origin requires, firstly and importantly, definitive pathologic confirmation of a normal appendix. Secondly, as published reports describe, IHC staining for SATB2. SATB2 is a protein with restricted expression to glandular cells of the lower gastrointestinal tract and frequent association with appendiceal mucinous neoplasms. Lastly, expression of CK20, CDX2 and CK7 that corroborates clinically in our patient with a PMP-derived ruptured appendiceal mucinous-like tumor arising from a secondary origin, a malignant transformed ovarian mature cystic teratoma. ${ }^{1,3}$

The decision to perform cytoreductive surgery is contingent upon disease biology, disease burden and performance status of the patient. In general, limited abdominopelvic peritoneal metastasis or low peritoneal cancer index $(\mathrm{PCl})$ with a low-grade tumor biology is a clinical presentation most amenable to this treatment option in healthy patients. ${ }^{14}$ Expert pathologic review is essential prior to proceeding to surgery. Intraoperatively, $\mathrm{PCl}$ is determined by scoring the size and/or confluence of disease and calculating the total score derived from 13 regions of the abdomen and pelvis (score range, $0-3$; total PCl range, 0-39). ${ }^{9}$ The principles of surgical cytoreduction of tumor is predicated upon six peritonectomy and visceral resective procedures that remove and/or strip cancer from peritoneal abdominopelvic and visceral surfaces as described by Sugarbaker. ${ }^{15}$ Importantly, the pharmacokinetic and clinical efficacy of heated intraperitoneal regional perfusion of chemotherapy or HIPEC, in part, relies upon successful removal of macroscopic disease and drug(s) employed. ${ }^{14}$ In our patient's case, a complete cytoreduction was performed and mitomycin $\mathrm{C}$, a potent alkylating chemotherapeutic agent with a high molecular weight (334 Daltons) conducive to intraperitoneal drug retention was utilized to provide an optimal therapeutic concentration time curve ratio with thermal temperature enhancement and tumor penetrance. $^{16}$ 
The HIPEC procedure is routinely carried out using a closed abdomen technique (Figure 3).

\section{Figure 3. Schematic of Hyperthermic Intraperitoneal Chemotherapy (HIPEC) Procedure. Printed with permission by Wilbur B. Bowne, MD.}

After cytoreduction of tumor, inflow and outflow drainage catheters connected to a perfusion pump are placed within the pelvis and upper abdomen, respectively. The patient's skin is closed around the catheters with accompanying temperature probes. Regional perfusion of crystalloid containing heated chemotherapy at moderate hyperthermia temperatures (40-44 degrees Celsius) is circulated and mechanically distributed throughout the abdomen and pelvis for a predetermined period of time in accord with the antineoplastic and chemotherapeutic properties of the agent(s) employed. At the conclusion, drainage catheters and chemotherapeutic perfusate are removed.

Historically, the first patient treated with hyperthermic intraperitoneal chemotherapy was in 1979 for recurrent PMP, having previously undergone CRS. Subsequent phase I and phase II clinical trials in the 1980s demonstrated effectiveness of intraperitoneal chemotherapy with improved survival in patients largely suffering from ovarian and appendiceal cancer. Currently, CRS and HIPEC is regarded as standard of care for the treatment of PMP. ${ }^{5}$ Recently, efficacy and survival benefit using HIPEC was reported by van Driel et al. when implemented as a treatment strategy during interval cytoreduction for stage III epithelial ovarian cancer in a multicenter, randomized, phase 3 trial. ${ }^{17}$ Moreover, this treatment approach has shown particular benefit for abdominal malignancies that include peritoneal mesothelioma, colorectal, and gastric carcinomas. ${ }^{14}$

Advanced abdominal malignancies, as in our patient, frequently present with significant tumor burden and upper abdominal, hepatobiliary involvement. There is little debate that suboptimal CRS offers no benefit to the patient. At our institution, cytoreductive surgery frequently includes extensive upper abdominal procedures involving consultant hepatobiliary, gastrointestinal (WBB, AN, $\mathrm{HL}$ ) along with gynecologic surgical oncologists (SDR, NGR) to optimize chance for complete macroscopic removal of tumor. ${ }^{8}$ As our patient report entails, PMP arising from a malignant transformed ovarian primary mature cystic teratoma is a rare 'diagnosis of exclusion'. As demonstrated in this current case, it initially masqueraded as an advanced gynecologic malignancy but on subsequent pathologic review resembled the DPAM-variant of PMP arising from a ruptured appendiceal-like neoplasm originating from a MCT. Importantly from a therapeutic standpoint, complete cytoreduction and HIPEC for the DPAM-variant of PMP has a 10 -year survival rate of $68 \% .{ }^{6}$ Specific experience applying CRS-HIPEC for malignant transformed ovarian teratomas with peritoneal dissemination, although rarely reported, describes potential treatment efficacy with modest disease recurrence and survival. ${ }^{10}$

Regardless of origin, PMP has a propensity to recur despite optimal therapy. Similar to traditional appendix-derived PMP, our patient's tumor harbored non-targetable mutant KRAS-GNAS co-mutations with ATM mutant positivity. ${ }^{18}$ Presence of these mutations indeed have a predominant biological impact. ${ }^{19}$ To date, current tumor grade pathologic classification systems for PMP (low grade in our 
patient) have yet shown to directly correlate with modern molecular biomarkers. Advances in molecular characterization of PMP will further enhance our understanding to predict tumor biology and improve identification of targetable, druggable therapeutic biomarkers to prevent disease recurrence. ${ }^{18}$

\section{Conclusion}

The present case represents an unusual occurrence and presentation of a rare clinical entity: a PMP syndrome arising in appendiceal-like tissue from a mature cystic teratoma. Discovery of a mature cystic teratoma with malignant transformations coincident with a PMP syndrome should promptly lead to an investigation into the malignancy status of the appendix. During surgery for removal of the mass and/or cytoreduction, the appendix should be removed and submitted for pathologic analysis. Notably, CRS and HIPEC are recommended treatments for appendiceal-derived PMP. However, the question will remain whether 'proof of concept' performance of CRS and HIPEC will ultimately contribute to similar durable disease control with PMP-derived from a transformed MCT. Our case provides a clinical clue and suggests that it does. This report adds to the existing evidence supporting this aggressive treatment option in these unique patients. Limitations of this case report include the retrospective nature of this review, duration of follow-up and requirement for more patients to validate this treatment strategy.

\section{Declarations}

\section{Acknowledgements}

Senior authors WBB and NGR wish to acknowledge the valuable assistance in the care of this patient provided by the surgical and gynecology oncology services at Thomas Jefferson University Hospital in the care of this patient.

\section{Authors' contributions}

FP, LK, MG, AG, JC, RL, SMC, SDR, AN, and HL contributed to the writing of the manuscript.

JC, WBB, and NGR contributed to the analysis of the patient's data/findings. All authors edited the final manuscript. All authors read and approved the final manuscript.

\section{Funding}

Not applicable (no funding received)

\section{Availability of data and material}

Data sharing is not applicable to this article as no datasets were generated or analyzed during the current study

\section{Ethics approval and consent to participate}


Not applicable

\section{Consent for publication}

Consent for publication was obtained from the patient and all co-authors. Written informed consent obtained from the patient.

\section{Competing interests}

The authors declare that they have no competing interests

\section{Author details}

${ }^{1}$ Department of Surgery, Thomas Jefferson University Hospital, 1100 Walnut Street, Suite 500, Philadelphia, PA 19107, USA. ${ }^{2}$ Department of Pathology, Thomas Jefferson University Hospital, 117 South $11^{\text {th }}$ Street, 204 Pavilion, Philadelphia, PA 19107, USA. ${ }^{3}$ Gynecologic Oncology, Thomas Jefferson University, 833 Chestnut Street \#1, Philadelphia, PA 19107, USA.

\section{References}

1. Yan, F., Shi, F., Li, X., Yu, C., Lin, Y., Li, Y., and Jin, Y. (2020). Clinicopathological characteristics of pseudomyxoma peritonei originated from ovaries. Cancer Management and Research, 12, 75697578, https://doi.org/10.2147/CMAR.S264474.

2. Ronnett, B., Kurman, R. J., Zahn, C. M., Shmookler, B. M., Jablonski, K. A., Kass, M. E., and Sugarbaker, P. H. (1995). Pseudomyxoma peritonei in women: A clinicopathologic analysis of 30 cases with emphasis on site of origin, prognosis, and relationship to ovarian mucinous tumors of low malignant potential. Human Pathology, 26(5), 509-524, https://doi.org/10.1016/0046-8177(95)90247-3.

3. Hwang, J. H., So, K. A., Modi, G., Lee, J. K., Lee, N. W., Lee, K. W., and Kim, I. (2009). Borderline-like mucinous tumor arising in mature cystic teratoma of the ovary associated with pseudomyxoma peritonei. International Journal of Gynecological Pathology, 28, 376-380, doi:

10.1097/PGP.0b013e318191e766.

4. Suh, D. S., Song, Y. J., Kwon, B. S., Lee, S., Lee, N. K., Choi, K. U., \& Kim, K. H. (2017). An unusual case of pseudomyxoma peritonei associated with synchronous primary mucinous tumors of the ovary and appendix: A case report. Oncology Letters, 13(6), 4813-4817. https://doi.org/10.3892/ol.2017.6079

5. Chicago Consensus Working Group. (2020). The Chicago Consensus on peritoneal surface malignancies: Management of appendiceal neoplasms. Cancer, 126(11), 2525-2533. https://doi.org/10.1002/cncr.32881

6. Chua, T. C., Moran, B. J., Sugarbaker, P. H., Levine, E. A., Glehen, O.,... Morris, D. L. (2012). Early and long term outcome data of patients with pseudomyxoma peritonei from appendiceal origin treated 
by a strategy of cytoreductive surgery and hyperthermic intraperitoneal chemotherapy. Journal of Clinical Oncology, 30(20), 2449-2456. DOI: 10.1200/JC0.2011.39.7166.

7. Moran, B. J., \& Cecil, T. D. (2003). The etiology, clinical presentation, and management of pseudomyxoma peritonei. Surgical Oncology Clinics of North America, 12(3), 585-603. https://doi.org/10.1016/s1055-3207(03)00026-7

8. Chi, D. S., Eisenhauer, E. L., Zivanovic, O., Sonoda, Y., Abu-Rustum, N. R.,... Barakat, R. R. (2009). Improved progression-free and overall survival in advanced ovarian cancer as a result of change in surgical paradigm. Gynecologic Oncology, 114, 26-31.

9. Jacquet, P., \& Sugarbaker, P. H. (1996). Clinical research methodologies in diagnosis and staging of patients with peritoneal carcinomatosis. Cancer Treatment and Research, 82, 359-374. https://doi.org/10.1007/978-1-4613-1247-5_23

10. Yu, H., Yonemura, Y., Hsieh, M., Wu, S., \& Shan, Y. (2019). Experience of applying cytoreductive surgery and hyperthermic intraperitoneal chemotherapy for ovarian teratoma with malignant transformation and peritoneal dissemination. Therapeutics and Clinical Risk Management, 2019(15), 129-136. https://doi.org/10.2147/TCRM.S190641.

11. Vang, R., Gown, A. M., Zhao, C., Barry, T. S., Isacson, C., Richardson, M. S., \& Ronnett, B. M. (2007). Ovarian mucinous tumors associated with mature cystic teratomas morphologic and immunohistochemical analysis identifies a subset of potential teratomatous origin that shares features of lower gastrointestinal tract mucinous tumors more commonly encountered as secondary tumors in the ovary. American Journal of Surgical Pathology, 31, 854-869, doi: 10.1097/PAS.0b013e31802efb45.

12. Morano, W. F., Gleeson, E. M., Sullivan, S. H., Padmanaban, V., Mapow, B. L., Shewokis, P. A., Esquivel, J., \& Bowne, W. B. (2018). Clinicopathological Features and Management of Appendiceal Mucoceles: A Systematic Review. The American Surgeon, 84(2), 273-281.

13. Padmanaban, V., Morano, W. F., Gleeson, E., Aggarwal, A., Mapow, B. L., Stein, D. E., \& Bowne, W. B. (2016). Incidentally discovered low-grade appendiceal mucinous neoplasm: A precursor to pseudomyxoma peritonei. Clinical Case Reports, 4(12), 1112-1116.

https://doi.org/10.1002/ccr3.694

14. Morano, W. F., Khalili, M., Chi, D. S., Bowne, W. B., \& Esquivel, J. (2017). Clinical studies in CRS and HIPEC: Trials, tribulations, and future directions - a systematic review. Journal of Surgical Oncology, $1-15$.

15. Sugarbaker, P. H. (1995). Peritonectomy procedures. Annals of Surgery, 221(1), 29-42.

16. Van der Speeten, K., Stuart, O. A., Chang, D., Mahteme, H., \& Sugarbaker, P. H. (2011). Changes induced by surgical and clinical factors in the pharmacology of intraperitoneal mitomycin $\mathrm{C}$ in 145 patients with peritoneal carcinomatosis. Cancer Chemotherapy and Pharmacology, 68(1), 147-156. https://doi.org/10.1007/s00280-010-1460-4

17. Van Driel, W. J., Koole, S. N., Sikorska, K., Schagen van Leeuwen, J. H., Schreuder, H. W. R.,... Sonke, G. S. (2018). Hyperthermic intraperitoneal chemotherapy in ovarian cancer. New England Journal of 
Medicine, 378(3), 230-240.

18. Gleeson, E. M., Feldman, R., Mapow, B. L., Mackovick, L. T., Ward, K. M., Morano, W. F., Rubin, R. R., \& Bowne, W. B. (2018). Appendix-derived pseudomyxoma peritonei (PMP): Molecular profiling toward treatment of a rare malignancy. American Journal of Clinical Oncology, 41(8), 777-783.

19. Pietrantonio, F., Perrone, F., Mennitto, A., Gleeson, E. M., Milione, M., Tamborini, E., Busico, A., Settanni, G., Berenato, R., Caporale, M., Morano, F., Bossi, I., Pellegrinelli, A., Di Bartolomeo, M., de Braud, F., Baratti, D., Bowne, W. B., Kusamura, S., \& Deraco, M. (2016). Toward the molecular dissection of peritoneal pseudomyxoma. Annals of Oncology: Official Journal of the European Society for Medical Oncology, 27(11), 2097-2103. https://doi.org/10.1093/annonc/mdw314

20. Takagi, H., Ichigo, S., Murase, T., Ikeda, T., \& Imai, A. (2012). Early diagnosis of malignant-transformed ovarian mature cystic teratoma: Fat-suppressed MRI findings. Journal of Gynecologic Oncology, 23(2), 125-128. https://doi.org/10.3802/jgo.2012.23.2.125

21. Chuang, H.-Y., Chen, Y.-T., Mac, T.-L., Chen, Y.-C., Chen, H.-S., Wang, W.-S., \& Tsai, E.-M. (2015). Urothelial carcinoma arising from an ovarian mature cystic teratoma. Taiwanese Journal of Obstetrics \& Gynecology, 54(4), 442-444. https://doi.org/10.1016/j.tjog.2015.06.003

22. Kudva, R., Ayachit, G. S., \& Ayachit, A. (2015). Malignant Melanoma Arising in an Ovarian Mature Cystic Teratoma-A Rare Entity. Journal of Clinical and Diagnostic Research: JCDR, 9(4), ED14ED16. https://doi.org/10.7860/JCDR/2015/12457.5817

23. Cokmez, H., Gulbahar, A., Yigit, S., \& Aydin, C. (2019). Oncocytic and tall columnar type papillary thyroid carcinoma arising on a mature cystic teratoma: A case report and literature review. JPMA. The Journal of the Pakistan Medical Association, 69(1), 116-119.

24. Chiu, H.-C., \& Chen, Y.-L. (2019). Trabecular carcinoid tumor arising from a mature cystic teratoma. $\mathrm{Ci}$ Ji Yi Xue Za Zhi = Tzu-Chi Medical Journal, 31(3), 192-194. https://doi.org/10.4103/tcmj.tcmj_91_18.

\section{Figures}




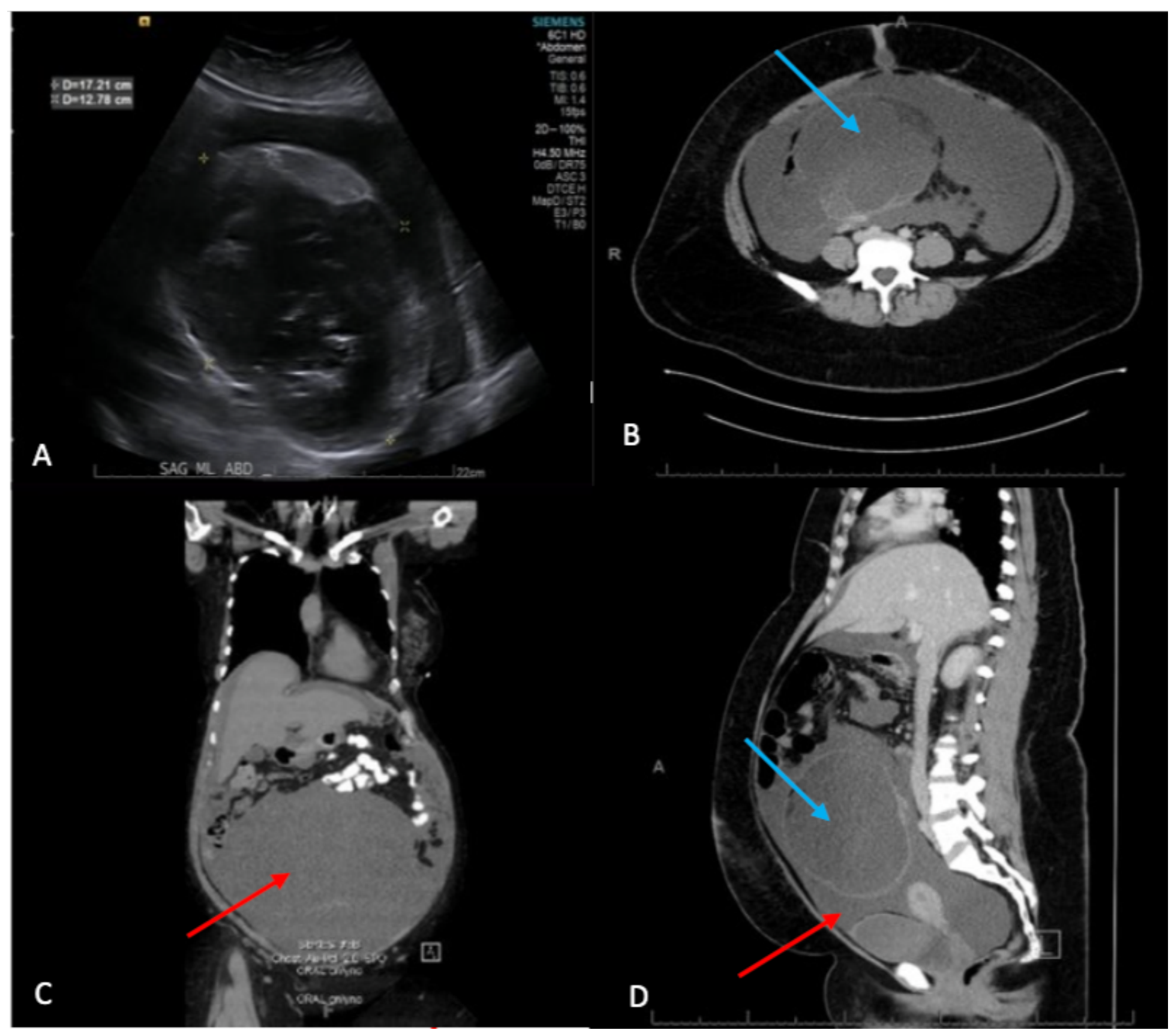

\section{Figure 1}

(A) Ultrasound images of the $18 \mathrm{~cm}$ mass (B-D) Contrast-enhanced perioperative abdominal CT scan of the $18 \mathrm{~cm}$ mass in the lower abdominal cavity $(B=$ axial, $C=$ coronal, $D=$ sagittal $)$. The imaging revealed a moderately large volume of mucinous ascites (red arrows) and a large right ovarian mass (blue arrows) concerning for cystic teratoma vs malignancy. 


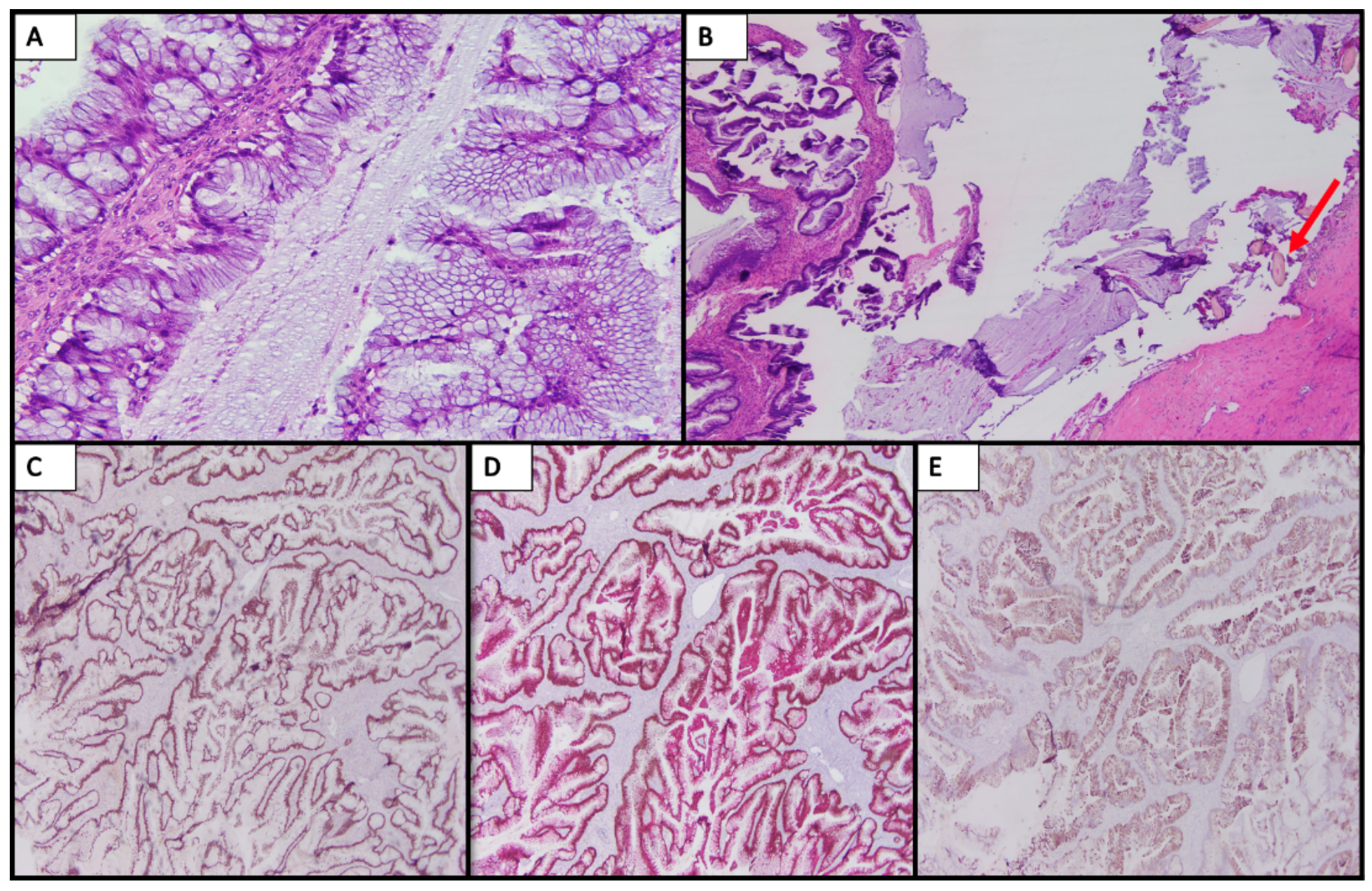

Figure 2

A: Mucinous neoplasm, right ovary, showing complex mucinous epithelium (200x); B: Hair follicles (red arrow) with adjacent mucinous neoplasm (40x); C. SATB2 showing strong nuclear staining (40x); D: CDX2/CK20 dual stain showing strong nuclear and cytoplasmic staining, respectively (40x); E. CK7 showing focal cytoplasmic staining. 


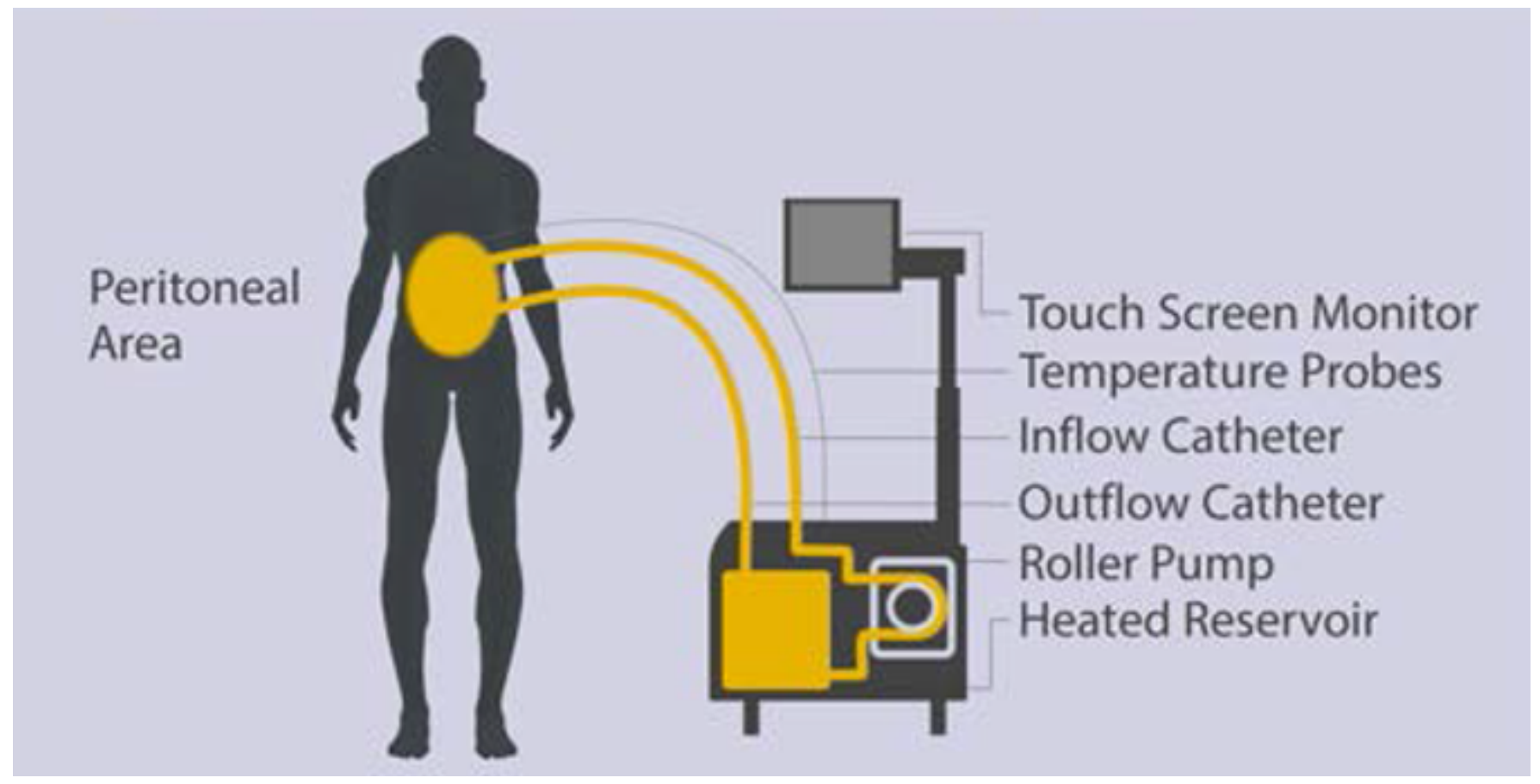

\section{Figure 3}

Schematic of Hyperthermic Intraperitoneal Chemotherapy (HIPEC) Procedure. Printed with permission by Wilbur B. Bowne, MD. 